\title{
Crescimento e desenvolvimento de filhos de mães adolescentes no primeiro ano de vida
}

\author{
Growth and development of children of adolescent mothers during the first year of life \\ Maria de Lourdes F. Vieira', Gladys Gripp Bicalho' ${ }^{2}$ João Luiz de C. P. Silva ${ }^{3}$, Antonio de Azevedo Barros Filho ${ }^{4}$
}

\section{RESUMO}

Objetivo: A gravidez e o parto na adolescência estão aumentando e suas conseqüências têm sido estudadas para identificar possíveis prejuízos para o desenvolvimento das crianças, ao longo dos primeiros anos de vida. O objetivo desta pesquisa foi estudar o crescimento e o desenvolvimento de filhos de mães adolescentes e compará-los com os de mães adultas.

Métodos: Foram selecionadas 122 crianças filhas de mães adolescentes e 123 de mulheres com 20 anos ou mais, nascidas no Centro de Atenção Integral à Saúde da Mulher da Universidade Estadual de Campinas, cujas idades variavam ao exame entre 11 e 13 meses de vida. Avaliaram-se variáveis maternas médicas e sociais: dados do parto, medidas antropométricas das crianças, necessidade de hospitalização no primeiro ano, utilização de creches no período e o desenvolvimento neuropsicomotor. Os dados foram analisados pelos programas SAS e SPSS. Foram aplicados os testes do qui-quadrado e análise de covariância, sendo significante $p<0,05$.

Resultados: Com um ano de idade, os filhos das adolescentes apresentaram peso e índice de massa corpórea menores que os controles, não sendo observadas diferenças no desenvolvimento neuropsicomotor.

Conclusões: Os filhos das adolescentes estudadas apresentaram desenvolvimento semelhante aos filhos de mães adultas, mostrando, entretanto, um deficit de crescimento a ser considerado.

Palavras-chave: gravidez na adolescência; crescimento; desenvolvimento infantil, antropometria.

\section{ABSTRACT}

Objective: Pregnancy among teenagers is increasingly frequent and its consequences need to be studied in order to identify possible problems regarding children's development during the first years of life. The aim of this study was to evaluate growth and development of infants born to teenager mothers compared to those born to adult mothers.

Methods: 122 infants of teenager mothers and 123 of adult mothers born at the maternity hospital of a university hospital at Campinas, São Paulo, Brazil, were enrolled. At study, children ages ranged from 11-13 months. Social and medical characteristics were analyzed: information regarding birth, anthropometric measures, need of hospitalization during the first year, use of day care centers, neurological and psychomotor development. Data were analyzed by SAS and SPSS softwares. Chi-square and analysis of covariance were applied, being significant a $p$ value $<0.05$.

Results: With one year of age, infants of teenager mothers had lower weight and body mass index than the controls. No differences among groups were noted regarding developmental milestones.

Conclusions: Children of teenager mothers have similar development but worse growth than those of adult mothers.

Key-words: pregnancy in adolescence; growth; child development; anthropometry.
1Professora doutora do Departamento de Pediatria da Faculdade de Medi-
cina da Universidade Federal de Alagoas, Maceió, AL, Brasil
2Professora doutora da Faculdade de Medicina da Pontifícia Universidade
Católica de Campinas, Campinas, SP, Brasil
3Professor titular de Obstetrícia do Departamento de Tocoginecologia da
Faculdade de Ciências Médicas da Universidade Estadual de Campinas
(FCM-Unicamp), Campinas, SP, Brasil
4Professor associado do Departamento de Pediatria e Pesquisador do Cen-
tro de Investigação em Pediatria da FCM-Unicamp, Campinas, SP, Brasil

Endereço para correspondência:

Gladys Gripp Bicalho

Avenida Lotário Teixeira, 215 - Parque Cidade Nova

CEP 13845-430 - Mogi Guaçu/SP

E-mail: gripp@dglnet.com.br

Recebido em: 18/6/2007

Aprovado em: 25/9/2007 


\section{Introdução}

A gestação e maternidade em mulheres adolescentes têm sido estudadas e discutidas em muitos aspectos, por se tratarem de situações de freqüência crescente, alvos de várias considerações ainda não bem estabelecidas ${ }^{(1)}$.

Dados do Ministério da Saúde informam que, em 2005, no Brasil, 22,7\% dos nascidos vivos eram filhos de mulheres com menos do que 20 anos $^{(2)}$. Tal freqüência, no mesmo ano, no Estado de São Paulo, foi $17 \%$ e, na cidade de Campinas, em São Paulo, $14,5 \%^{(2)}$. Historicamente, não se trata de um evento novo; no entanto, preocupa os responsáveis pela saúde materno-infantil no que diz respeito aos resultados gestacionais e aos cuidados que tais mães seriam capazes de destinar aos seus filhos.

As adolescentes têm características biológicas compatíveis com um desempenho obstétrico satisfatório ${ }^{(3,4)}$ e a gestação no início da vida reprodutiva pode exercer efeito protetor sobre o crescimento intra-uterino, quando os fatores desfavoráveis são controlados ${ }^{(3)}$. No entanto, como a vida das adolescentes é ligada a várias carências, observa-se que seus recém-nascidos apresentam maior freqüência de baixo peso ao nascer, especialmente por prematuridade, com conseqüências imediatas e em longo prazo $^{(5)}$. Algumas pesquisas sugerem que os filhos de adolescentes mostram atraso no desenvolvimento ${ }^{(6,7)}$, maior proporção de problemas psicológicos, além de deficiência de crescimento, maior morbidade e mortalidade infantil, quando comparados as filhos de mulheres adultas ${ }^{(8-11)}$.

Há um risco comportamental durante a adolescência, que ultrapassa os critérios biomédicos e atinge variáveis sociais $^{(12)}$. Provavelmente, a adolescente que está exposta a fatores desfavoráveis durante a gestação continuará vivendo as mesmas situações após o parto, o que resultaria em problemas para o seu filho. Apesar disto, não se observam diferenças pela procura pelos serviços de puericultura e, nas dificuldades iniciais com a amamentação, a intenção de realizá-la ${ }^{(13)}$ e a sua prática, sugerindo que as adolescentes são tão cuidadosas com seus filhos como as mães de mais idade ${ }^{(14,15)}$.

Embora a adolescente seja considerada freqüentemente incapaz de cuidar de uma criança, há controvérsias quanto ao resultado final do seu desempenho e as conseqüências para o seu filho ${ }^{(16)}$. Considerando que a saúde do lactente sofre influências dos cuidados fornecidos pela mãe, esta pesquisa objetivou estudar o crescimento e o desenvolvimento de filhos de adolescentes e compará-los com os de mulheres adultas.

\section{Métodos}

Foram estudadas 245 crianças, 122 de mães adolescentes (menos de 20 anos) e 123 de adultas (com 20 anos ou mais), nascidas no Centro de Atenção Integral à Saúde da Mulher da Universidade Estadual de Campinas (Unicamp), entre 1998 e 1999. Todas as crianças nasceram a termo, com peso maior ou igual a $2.500 \mathrm{~g}$, de gestações únicas e mães primigestas; foram excluídas as que haviam sido classificadas como pequenas para a idade gestacional.

Tratou-se de um estudo transversal realizado durante os anos de 1999 e 2000, que incluiu crianças com idade entre 11 e 13 meses. As informações foram resgatadas do prontuário médico e obtidas por consulta da criança e entrevista com a mãe no domicílio da família ou no Centro de Investigações em Pediatria da Unicamp, após convocação específica.

Foram estudados dois grupos de mães: as adolescentes de idades abaixo de 20 anos, e as adultas, que tinham entre 20 e 30 anos. Ao se comparar as adolescentes com idades $\leq 16$ anos com aquelas entre 17 e 19 anos, não se observou diferença estatisticamente significativa com relação ao peso, comprimento e perímetro cefálico (PC) de seus filhos ao nascer ( $p=0,098,0,107$ e 0,697, respectivamente). O mesmo ocorreu quando as crianças tinham um ano de idade ( $p=0,272,0,820$ e 0,505 , respectivamente). Desta forma, todos os filhos de adolescentes foram analisados em um só grupo.

As variáveis maternas estudadas foram: situação conjugal ao engravidar e quando o filho completou um ano, classificada como unida (casada ou amasiada) ou não (solteira, separada, desquitada ou viúva); escolaridade (número de séries completas), que foi definida quanto à adequação para idade, levando-se em conta que aos sete anos a criança deveria estar cursando a primeira série do ensino fundamental; trabalho materno (fora de casa ou no lar); número de consultas durante o pré-natal (adequado, quando $\geq 6$ ); e tipo de parto (vaginal ou cesárea).

Das crianças, foram avaliados os seguintes dados: Apgar ao quinto minuto (sendo $\geq 7$ indicativo de boas condições de nascimento); peso, comprimento, PC e adequação de peso ao nascer para a idade gestacional. Com um ano de idade, estudaram-se peso, comprimento, PC, perímetro braquial, índice de massa corpórea $\left(\mathrm{IMC}=\right.$ peso/comprimento $\left.{ }^{2}\right)$, pregas cutâneas tricipital e subescapular, hospitalização no primeiro ano; freqüência a creches e desenvolvimento neuropsicomotor, utilizando parte da escala de Denver ${ }^{(17)}$. 
O tamanho da amostra foi calculado considerando o erro alfa de $5 \%$ e o beta de $10 \%$, usando as variáveis de interesse (peso, comprimento e PC): ao menos 102 crianças deveriam ser estudadas em cada grupo. Os dados foram digitados em SPSS (versão 10.0) e analisados pelo SAS (versão 6.12). O teste do qui-quadradoe o exato de Fisher foram utilizados para comparar proporções entre os grupos, considerando-se significante $p<0,05$. $\mathrm{O}$ valor de $p$ apresentado para as medidas antropométricas dos recém-nascidos foi calculado com base nas médias corrigidas pelo sexo, aplicando-se a análise de covariância (ANCOVA).

A pesquisa foi realizada após a aprovação do Comitê de Ética em Pesquisa da Unicamp e assinatura do termo de consentimento livre e esclarecido pela mãe da criança.

\section{Resultados}

Foram estudados 122 filhos de mães adolescentes e 123 de mães adultas. A média de idade materna foi de 17,0 $\pm 1,6$ anos no primeiro grupo e $23,2 \pm 2,7$ no segundo. A Tabela 1 apresenta as características maternas de ambos os grupos. Entre as mães adultas, foram observadas freqüências maiores de consultas pré-natais $(p=0,024)$, trabalho fora de casa $(p=0,018)$ e presença de um companheiro fixo $(p=0,001)$. Foi identificada a mudança na situação conjugal das adolescentes de não unidas ao engravidar para unidas, quando a criança atingiu um ano de idade $(p=0,01)$.
A Tabela 2 apresenta as características e medidas de nascimento das crianças estudadas. A idade gestacional média não diferiu entre os grupos: observou-se $39,6 \pm 1,1$ para os filhos das adolescentes e 39,7 $\pm 1,1$ para os filhos das adultas. A presença de boletim de Apgar foi maior ou igual a sete no quinto minuto também em ambos os grupos (98\%). Mais recém-nascidos filhos de adolescentes eram adequados para a idade gestacional $(p=0,042)$.

As medidas antropométricas realizadas com um ano de idade indicam diferença significativa para o peso e IMC entre os grupos (Tabela 3). A freqüência a creches no primeiro ano de vida foi relatada por $7 \%$ das mães adolescentes e $19 \%$ das não-adolescentes $(p=0,005)$. Não houve diferença, estatística quanto à freqüência de hospitalização das crianças estudadas filhas das adolescentes (17\%) ou de adultas $(15 \% ; p=0,581)$.

A Tabela 4 mostra que os filhos de mães adolescentes e de adultas não são estatisticamente diferentes com relação ao desenvolvimento neuropsicomotor, o qual foi adequado para a idade na maioria dos casos. As crianças dos dois grupos apresentaram a mesma moda para os seguintes resultados do desenvolvimento: um mês para sorrir espontaneamente, três meses para segurar o pescoço, seis meses para sentar sem apoio, dez meses para ficar de pé sem apoio e andar com ajuda e 12 meses para andar sem apoio.

Tabela 1 - Características de mães adolescentes e adultas estudadas

\begin{tabular}{lccc}
\hline & $\begin{array}{c}\text { Adolescentes } \\
\text { N (\%) }\end{array}$ & $\begin{array}{c}\text { Adultas } \\
\text { N (\%) }\end{array}$ & Valor de $\boldsymbol{p}$ \\
\hline Pré-natal $\geq 6$ consultas & $93(77 \%)$ & $109(89 \%)$ & 0,002 \\
Escolaridade adequada para a idade & $32(26 \%)$ & $44(36 \%)$ & 0,106 \\
Trabalho materno fora de casa & $27(22 \%)$ & $44(36 \%)$ & 0,018 \\
Parto cesáreo & $35(29 \%)$ & $41(34 \%)$ & 0,599 \\
Situação conjugal ao engravidar=unida & $58(48 \%)$ & $93(76 \%)$ & 0,001 \\
Situação conjugal unida com filho de um ano & $89(73 \%)$ & $106(86 \%)$ & 0,010 \\
\hline
\end{tabular}

Tabela 2 - Características neonatais de filhos de adolescentes e mulheres adultas incluídos no estudo

\begin{tabular}{lccc}
\hline & $\begin{array}{c}\text { Mães adolescentes } \\
\mathbf{N = 1 2 2}\end{array}$ & $\begin{array}{c}\text { Mães adultas } \\
\mathbf{N = 1 2 3}\end{array}$ & Valor de $\boldsymbol{p}$ \\
\hline Sexo masculino & $63(52 \%)$ & $60(49 \%)$ & 0,655 \\
Peso (g) & $3.227 \pm 363$ & $3.291 \pm 429$ & 0,169 \\
Comprimento $(\mathrm{cm})$ & $49,2 \pm 1,7$ & $49,7 \pm 2,0$ & 0,027 \\
Perímetro cefálico $(\mathrm{cm})$ & $34,3 \pm 1,3$ & $34,4 \pm 1,5$ & 0,647 \\
Peso adequado para idade gestacional & $116(95 \%)$ & $108(88 \%)$ & 0,042 \\
\hline
\end{tabular}


Tabela 3 - Características de filhos de mães adolescentes e adultas com um ano de idade.

\begin{tabular}{lccc}
\hline & $\begin{array}{c}\text { Mães adolescentes } \\
\mathbf{N = 1 2 2}\end{array}$ & Mães adultas & \multirow{2}{*}{ Valor de $\boldsymbol{p}$} \\
\hline Peso $(\mathrm{g})$ & $9.813 \pm 1.095$ & $10.138 \pm 1.186$ & 0,019 \\
Comprimento $(\mathrm{cm})$ & $76,8 \pm 3,0$ & $77,0 \pm 2,7$ & 0,394 \\
Perímetro cefálico $(\mathrm{cm})$ & $46,3 \pm 1,2$ & $46,5 \pm 1,3$ & 0,184 \\
Perímetro braquial $(\mathrm{cm})$ & $15,4 \pm 1,1$ & $15,7 \pm 1,2$ & 0,120 \\
Prega tricipital $(\mathrm{cm})$ & $8,6 \pm 2,1$ & $9,0 \pm 2,1$ & 0,130 \\
Prega subescapular $(\mathrm{mm})$ & $6,8 \pm 1,7$ & $6,9 \pm 1,7$ & 0,589 \\
$\mathrm{IMC}\left(\mathrm{kg} / \mathrm{m}^{2}\right)$ & $16,5 \pm 1,4$ & $16,9 \pm 1,5$ & 0,041 \\
\hline
\end{tabular}

Tabela 4 - Desenvolvimento neuropsicomotor dos filhos de mães adolescentes e de adultas com um ano de idade

\begin{tabular}{|c|c|c|c|}
\hline & $\begin{array}{c}\text { Mães adolescentes } \\
\mathrm{N}=122\end{array}$ & $\begin{array}{l}\text { Mães adultas } \\
\mathrm{N}=123\end{array}$ & Valor de $p$ \\
\hline Motricidade fina adequada & $120(98 \%)$ & $120(98 \%)$ & 1,000 \\
\hline Bate dois cubos que segura na mão & $107(88 \%)$ & $109(89 \%)$ & \\
\hline Pega objetos com polegar e indicador & $120(98 \%)$ & $120(98 \%)$ & \\
\hline Motricidade grosseira adequada & $121(99 \%)$ & $120(98 \%)$ & 0,622 \\
\hline Anda com apoio & $122(100 \%)$ & $122(99 \%)$ & \\
\hline Anda sem apoio & $100(82 \%)$ & $92(75 \%)$ & \\
\hline Abaixa-se e levanta-se & $121(99 \%)$ & $120(98 \%)$ & \\
\hline Socialização adequada & $120(98 \%)$ & $121(98 \%)$ & 1,000 \\
\hline Joga bola & $115(94 \%)$ & $117(95 \%)$ & \\
\hline Bebe de copo ou xícara & $105(86 \%)$ & $112(91 \%)$ & \\
\hline Brinca de espiar/esconder & $120(98 \%)$ & $122(99 \%)$ & \\
\hline Linguagem adequada & $116(95 \%)$ & $122(99 \%)$ & 0,066 \\
\hline Fala "papá" ou "mamã" & $115(94 \%)$ & $121(98 \%)$ & \\
\hline Fala três ou mais palavras & $107(88 \%)$ & $116(94 \%)$ & \\
\hline
\end{tabular}

\section{Discussão}

As crianças analisadas apresentaram o mesmo desempenho neuropsicomotor ao final do primeiro ano de vida, não havendo diferença quando se tratava de filhos de adolescentes ou adultas. $\mathrm{O}$ crescimento em peso e o IMC foram maiores no segundo grupo, mas a diferença é pequena e não tem representação clínica.

As crianças filhas de adolescentes não só mostraram um desenvolvimento semelhante ao dos filhos de adultas, como também o fizeram de maneira adequada, acima de $90 \%$. Os resultados poderiam ter sido favorecidos devido à média de idade materna ter sido de 17,0土1,6 anos, pois, para alguns autores, os riscos se concentrariam nas idades menores, de 13 a $17 \operatorname{anos}^{(18)}$.

Os testes habitualmente utilizados para a avaliação inicial do desenvolvimento neuropsicomotor são questionáveis por suas limitações e, principalmente, por perderem informações sobre a sua variabilidade ${ }^{(19)}$. No entanto, o exame aplicado neste estudo é recomendado habitualmente como um dos instrumentos de triagem para avaliar o desenvolvimento infantil aos 12 meses de idade e tal instrumento indicou um bom desempenho neuropsicomotor das crianças estudadas.

Resultados favoráveis sobre a maternidade no início da vida reprodutiva têm sido relatados e sugerem que os filhos das adolescentes são até mesmo beneficiados por esta condição, podendo apresentar média de idade mais precoce para alguns marcadores do desenvolvimento, quando comparados aos filhos de adultas ${ }^{(16)}$. No entanto, há controvérsias, visto que a gestação na adolescência é acompanhada habitualmente de inseguranças e vários problemas que não se resolvem em curto prazo. A pouca profissionalização e a baixa renda e escolaridade dos pais - particularmente da mãe $e^{(13,20)}-$, são fatores que interferem no crescimento das crianças, podendo torná-lo 
insatisfatório ${ }^{(21)}$. Tratam-se de limitações psicossociais que influenciam no assumir a maternidade e na formação do vínculo mãe/filho, não permitindo adequado fortalecimento $^{(3)}$. Considera-se que poderia haver maior dificuldade da adolescente em desenvolver ligações afetivas com seu filho, o que, somado à imaturidade, baixa auto-estima e ter um filho numa situação conflituosa, poderia resultar em desvantagens para o desenvolvimento da criança ${ }^{(22)}$.

De maneira similar à de outras pesquisas, observou-se que as adolescentes estudadas foram expostas a situações sociais desprivilegiadas por serem menos unidas a parceiro fixo e por terem apresentado menor freqüência de trabalho regular ${ }^{(12,23)}$. Por outro lado, tal situação poderia ser compensada por algum ganho particular em razão de sua condição de risco e vulnerabilidade social ou de ajuda financeira solidária ou institucional, beneficiando suas famílias. Nesta pesquisa, os resultados mostraram que estas crianças tiveram menos necessidade de freqüentar creches, sugerindo que puderam ficar mais tempo em companhia e cuidados de suas mães ou círculo parental.

Historicamente, a mudança no papel social da mulher, que aumenta suas atividades e trabalho fora de casa, pode privar a criança dos cuidados maternos, tão importantes no primeiro ano de vida. As mães adolescentes, quase crianças, seriam incapazes de cuidar de seus filhos, resultando em um círculo vicioso que poderia se perpetuar por várias gerações ${ }^{(22)}$. As transformações sociais, que incorporaram a mulher cada vez mais ao mercado de trabalho, tornam a utilização de creches mais freqüente e necessária.

As creches configuram maior risco para doenças respiratórias e de natureza infecto-contagiosas, o que, para alguns autores, indica que os bebês devam permanecer em casa, onde o ambiente restrito não os exporia a um grande número de contactantes ${ }^{(24)}$. Nesta amostra, demonstra-se que as adolescentes ficaram mais tempo com seus filhos que as mães adultas, provavelmente por sua condição de menor inserção escolar e empregatícia, beneficiando-os não só por evitar a creche, mas também por facilitar o vínculo afetivo na família.

O padrão de interação mãe/filho é importante para a saúde mental da criança. As privações cultural, financeira, educacional e afetiva potencializam-se e podem resultar em deficits no desenvolvimento intelectual e emocional da criança ${ }^{(22)}$. Admite-se, portanto, que o padrão de crescimento e desenvolvimento resulte de interação multifatorial e a idade materna pode não estar sendo a responsável pelo seu comprometimento $^{(18)}$.
$\mathrm{Na}$ amostra estudada, não houve diferença quanto à média do peso ao nascer dos dois grupos e os filhos das adolescentes cresceram de forma mais adequada para a idade gestacional. Estes resultados são semelhantes aos que vêm sendo obtidos por outras pesquisas que sugerem que a gestação na adolescência possa exercer um efeito protetor sobre o crescimento intra-uterino ${ }^{(4)}$. Os resultados confirmam a observação de que as adolescentes freqüentam menos os serviços de prénatal ${ }^{(25)}$, mas, entre as crianças estudadas, este fato não parece ter prejudicado o peso ao nascer.

No final do primeiro ano de vida, os grupos diferenciaram-se por menor peso e IMC das crianças filhas das adolescentes; no entanto, esta diferença foi muito pequena do ponto de vista biológico. Observou-se maior freqüência de hospitalização entre os filhos das adolescentes, mas não houve diferença significativa em relação à dos filhos de mães adultas.

Um programa de vigilância à saúde infantil de Belo Horizonte não evidenciou maiores agravos nos filhos de adolescentes e o padrão de crescimento não se modificou, quando comparado aos filhos de mães adultas. Embora a condição de filho de mãe adolescente seja considerada um critério de risco de morbimortalidade infantil, a idade materna não tem sido responsabilizada isoladamente por resultados desfavoráveis ${ }^{(18)}$. O baixo peso ao nascer por prematuridade, que tem maior frequiência em filhos das adolescentes, parece ser um dos responsáveis pelos desfechos desfavoráveis ${ }^{(18)}$. As adolescentes estudadas em Belo Horizonte fizeram acompanhamento pré-natal, continuaram a morar com seus pais após o parto e contavam com a ajuda de seus familiares, o que provavelmente contribuiu para minimizar o risco de morbimortalidade de suas crianças. Tais resultados sugerem que a idade materna não teria influência no desfecho, se o contexto da vida da adolescente não fosse composto de uma somatória de fatores de risco que a expõe durante a gestação e após o parto.

O perfil desfavorável das mães adolescentes pode ter sido minimizado pela mudança significativa da situação conjugal para casada ou amasiada no período de estudo. Este suporte familiar é reconhecido como facilitador de um bom desempenho do crescimento e desenvolvimento infantil ${ }^{(26)}$. A família é considerada a entidade social básica e ter uma família bem estruturada constitui fator de proteção para o desenvolvimento e o crescimento da criança. Muitas mães adolescentes continuam morando com seus pais após o parto e contam com a ajuda de seus familiares ${ }^{(20)}$, o que contribui para minimizar o risco de doenças no primeiro ano de vida de seus filhos ${ }^{(18)}$. 
Habitualmente, existe a expectativa de que a adolescente não terá filhos saudáveis e que, para atingir este resultado, deveria se transformar primeiramente em adulta. A pergunta que se coloca é se esta condição é absolutamente necessária ou poderia ser modificada se a adolescente fosse apoiada apropriadamente pelos serviços comunitários, por sua família e por programas de saúde, e se recebesse suporte suficiente para desempenhar a maternidade de forma adequada. A gravidez na adolescência nem sempre é percebida como um problema, especialmente quando há aceitação e apoio familiar, destacando-se a participação das avós maternas ${ }^{(27)}$.

Os resultados desta pesquisa sugerem que a adolescente é capaz de cuidar com sucesso de seu filho. Para tanto, a assistência deve ser estabelecida de modo abrangente para o binômio mãe/fillho e não só direcionada à mãe no pré-natal e à criança após o parto. Há vários aspectos difíceis de intervenção imediata, como os problemas relacionados às desigualdades

\section{Referências bibliográficas}

1. Spitz AM, Velebil P, Koonin LM, Strauss LT, Goodman KA, Wingo P et al. Pregnancy, abortion, and birth rates among US adolescents--1980, 1985, and 1990. JAMA 1996;275: 989-94.

2. Brasil - Ministério da Saúde - DATASUS [homepage on the Internet]. Informações de Saúde - Estatísticas Vitais - Mortalidade e Nascidos Vivos: nascidos vivos desde 1994 [cited 2007 Sep 19]. Available from: http://tabnet.datasus. gov.br/cgi/deftohtm.exe?sinasc/cnv/nvuf.def

3. Magalhães ML, Furtado FM, Nogueira MB, Carvalho FH, Almeida FML, Mattar $\mathrm{R}$ et al. Gestação na adolescência precoce e tardia - há diferença nos riscos obstétricos? Rev Bras Ginecol Obstet 2006;28:446-52.

4. Mariotoni GG, Barros Filho AA. A gravidez na adolescência é fator de risco para o baixo peso ao nascer? J Pediatr (Rio J) 1998;74:107-13.

5. Puffer RR, Serrano CV. Patterns of birthweights: scientific publication $n^{\circ} 504$. Washington: PAHO; 1987.

6. Romero MI, Yetzen G, Molina R, Alarcón G, González E, Arestizábal E et al. Caracteristicas del primer año de vida de hijos de madres adolescentes: un estudio descriptivo de seguimiento. Cuad Med-Soc (Santiago de Chile) 1984;25:124-9

7. Belitzky R, Cruz CA, Marinho E, Tenzer SM. Resultados perinatales en madres jóvenes: estudio comparativo en maternidades latinoamericanas - publicación científica 489. Washington: OPAS; 1986.

8. Molina R, Romero MI. El embarazo en la adolescencia: la experiencia chilena - publicación científica 489. Washington: OPAS; 1986.

9. Valenzuela MS, Abarca BC, Aillach EB, Aliste VC, Alvarado CS, Bocic GA et al. Primer año de vida en hijos de adolescentes. Bol Hosp San Juan de Dios 1987;34:81-5.

10. Davis S. Gravidez em adolescentes. Pediatr Clin North Am 1989;3:691-707.

11. Esperidião S, Júlio AG, Marcolim M, Silva NA, Oliverio IA, Nascimento JP et al. Avaliação dos recém-nascidos de mães adolescentes no Hospital Municipal de Santo André. Correlação com algumas variáveis maternas. Pediatr Mod 1992;28:526-9.

12. Feijó RB, Oliveira EA. Comportamento de risco na adolescência. J Pediatr (Rio J) 2001;77(Suppl 2):S125-34

13. Chalem E, Mitsuhiro SS, Ferri CP, Barros MC, Guinsburg R, Laranjeira R. Gravidez na adolescência: perfil sócio-demográfico e comportamental de uma população da periferia de São Paulo, Brasil. Cad Saúde Pública 2007;23:177-86. socioeconômicas que levam à necessidade de buscar recursos para o sustento próprio e do filho. Assim, o esforço dos profissionais envolvidos deve ser no sentido de resgatar aspectos educacionais, psicológicos e sociais, além de fornecer atenção diferenciada para com a saúde do binômio.

Embora a gestação na adolescência não deva ser incentivada, aquela parece não prejudicar o primeiro ano de vida da criança quanto ao crescimento e desenvolvimento. No entanto, é recomendável que a adolescente receba uma assistência especial, de modo multidisciplinar e interdisciplinar, na qual a censura preconceituosa não aconteça de modo sistemático e haja oportunidade de a adolescente aproximar-se dos profissionais que a assistem, trazendo suas dúvidas, inseguranças e preocupações, para desenvolver a responsabilidade de cuidar de seu filho de forma suficiente ${ }^{(28)}$. Este apoio tem sido sugerido desde a gestação, por proteger o feto e resultar em benefícios que podem se estender aos primeiros anos de vida da criança.

14. Vieira MLF, Silva JL, Barros Filho AA. A amamentação e a alimentação complementar de filhos de mães adolescentes são diferentes das de filhos de mães adultas? J Pediatr (Rio J) 2003;79:317-24.

15. Frota DAL, Marcopito LF. Amamentação entre mães adolescentes e nãoadolescentes, Montes Claros, MG. Rev Saúde Pública 2004;38:85-92.

16. Carruth BR, Nevling W, Skinner JD. Developmental and food profiles of infants born to adolescent and adult mothers. J Adolesc Health 1997;20: 434-41.

17. Frankenburg WK, Dodds J, Archer P, Shapiro H, Bresnick B. The Denver II: a major revision and restandardization of Denver Developmental Screening Test. Pediatrics 1992;89:91-7.

18. Goulart LM, Xavier CC, Goulart EM, Somarriba MG, Almeida CG, Costa AL. Avaliação da ação de vigilância à morbi-mortalidade infantil na periferia de Belo Horizonte, Minas Gerais. Rev Bras Saude Mater Infant 2003;3:291-304.

19. Drachler ML. Medindo o desenvolvimento infantil em estudos epidemiológicos: dificuldades subjacentes. J Pediatr 2000;76:401-3.

20. Dias $A B$, Aquino EM. Maternidade e paternidade na adolescência: algumas constatações em três cidades do Brasil. Cad Saúde Pública 2006;22:1447-58.

21. Antonio MA, Morcillo AM, Piedrabuena AE, Carniel EF. Análise do perfil de crescimento de 566 crianças com idade entre 3 meses e 3 anos matriculadas nas 14 creches municipais de Paulínia (SP). J Pediatr (Rio J) 1996;72:245-50.

22. Campos AL, Nascimento CF, Grazini JT, Assis AN, Vitolo MR, Nóbrega FJ. Aspectos nutricionais, psicológicos e sociais de mães de crianças desnutridas. J Pediatr (Rio J) 1995;71:214-8.

23. Muller RC, Barros Filho AA. Considerações e reflexões sobre a atenção à saúde do adolescente. Rev Bras Saúde Esc 1996;4:31-40.

24. Amorim KS, Rossetti-Ferreira MC. Análise crítica de investigações sobre doenças infecciosas respiratórias em crianças que freqüentam creche. J Pediatr (Rio J) 1999;75:313-20.

25. Mirkin SR, Leon RF, Frau D. Embarazo en adolescentes. "Fenómeno potenciado". Obst Y Ginec Lat Americ 1994;52:115-28.

26. Unger DG, Cooley M. Partner and grandmother contact in black and white teen parent families. J Adolesc Health 1992;13:546-52.

27. Lima CT, Feliciano KV, Carvalho MF, Souza AP, Menabó JB, Ramos LS et al. Percepções e práticas de adolescentes grávidas e de familiares em relação à gestação. Rev Bras Saude Matern Infant 2004;4:71-83.

28. Madeira AMF. Assistindo a adolescente e seu filho em uma unidade básica de saúde. Rev enfermagem UERJ 1999;7:173-7. 